\title{
A simulation-based approach to study the influence of different production flows on manufacturing of customized products
}

\author{
Żywicki, K. ${ }^{\mathrm{a},}{ }^{*}$, Rewers, P. ${ }^{\mathrm{a}}$ \\ ${ }^{\mathrm{a}}$ Faculty of Mechanical Engineering, Poznan University of Technology, Poznan, Poland
}

\section{A B S TRACT}

Manufacturing products tailored to the individual requirements of customers is a must if companies want to compete effectively on the market. The production of customized goods poses new challenges for all areas of functioning of production systems. It is necessary to adopt such rules and methods that will allow a flexible response to product design changes and their demand In the organization of production flow (materials and information). The article presents research carried out in the SmartFactory laboratory of the Poznań University of Technology regarding the impact of the structure of products (customization) on the realization of current production orders. The research was carried out using the FlexSim simulation environment. Based on simulation experiments for three forms of organization of production flow with varying degrees of flexibility of production resources, an analysis was made of the time of execution of various sets of production orders and the level of use of available working time. The results of research indicate that in the production of products with low and high planned labor consumption, the use of universal production station is the most advantageous. For such a solution, the degree of utilization of the available working time of production stations is also the highest. It was also found that the principles of scheduling production orders affect the effectiveness of the production system. The best results were obtained for the production schedule, where the sequence of production orders was established from the lowest planned time of resource loading.
\end{abstract}

\section{ARTICLE INFO}

Keywords:

Smart manufacturing;

Production flow;

Customized products;

Variant products;

Discrete-event simulation (DES);

FlexSim simulation modeling and

analysis software

*Corresponding author:

krzysztof.zywicki@put.poznan.pl

(Żywicki, K.)

Article history:

Received 26 May 2020

Revised 4 December 2020

Accepted 7 December 2020

\section{References}

[1] Liker, J.K., Meier, D. (2005). The Toyota way fieldbook: A practical guide for implementing Toyota's 4Ps, 1st edition, McGraw-Hill, London, United Kingdom.

[2] Wang, Y., Ma, H.-S., Yang, J.-H., Wang, K.-S. (2017). Industry 4.0: A way from mass customization to mass personalization production, Advances in Manufacturing, Vol. 5, 311-320, doi: 10.1007/s40436-017-0204-7.

[3] Davis, S.M. (1987). Future perfect, Addison-Wesley Publishing, Boston, USA.

[4] Pine II, B.J. (1993). Mass customization: The new frontier in business competition, Harvard Business School Press, Boston, USA.

[5] Kamble, S., Gunasekaran, A., Dhone, N.C. (2020). Industry 4.0 and lean manufacturing practices for sustainable organisational performance in Indian manufacturing companies, International Journal of Production Research, Vol. 58, No. 5, 1319-1337, doi: 10.1080/00207543.2019.1630772.

[6] Tseng, M.M., Jiao, J., Merchant, M.E. (1996). Design for mass customization, CIRP Annals, Vol. 45, No. 1, 153-156, doi: 10.1016/S0007-8506(07)63036-4.

[7] Gilmore, J.H, Pine II, B.J. (1997). The four faces of mass customization, Harvard Business Review, Vol. 75, No. 1, 91-101. 
[8] Tian, X., Huang, L., Jia, X., Zhang, Z. (2008). Exploring parameterised process planning for mass customisation, In: Yan, X.T., Jiang, C., Eynard, B. (eds.), Advanced Design and Manufacture to Gain a Competitive Edge, Springer, London, United Kingdom, 643-652, doi: 10.1007/978-1-84800-241-8 65.

[9] Żywicki, K., Zawadzki, P., Hamrol, A. (2017). Preparation and production control in smart factory model, In: Rocha, Á., Correia, A., Adeli, H., Reis, L., Costanzo, S. (eds.), Recent advances in information systems and technologies, WorldCIST 2017. Advances in Intelligent Systems and Computing, Vol. 571, Springer, Cham, Switzerland, 519527, doi: 10.1007/978-3-319-56541-5_53.

[10] Ko, E., Kincade, D., Brown, J.R. (2000). Impact of business type upon the adoption of quick response technologies - The apparel industry experience, International Journal of Operations \& Production Management, Vol. 20, No. 9, 1093-1111, doi: 10.1108/01443570010339172.

[11] Lebosse, S., Taghipour, A., Canel-Depitre, B. (2017). Quick response to fluctuations in supply chains: A review, Journal of Advanced Management Science, Vol. 5, No. 5, 394-400, doi: 10.18178/joams.5.5.394-400.

[12] Tseng, M.M., Wang, Y., Jiao, R.J. (2017). Mass customization, In: Laperrière, L., Reinhart, G. (eds.), CIRP Encyclopedia of Production Engineering, Springer, Berlin, Germany, doi: 10.1007/978-3-642-35950-7 16701-3.

[13] Schwab, K. (2017). The fourth industrial revolution, World Economic Forum, New York, USA.

[14] Żywicki, K., Zawadzki, P., Górski, F. (2018). Virtual reality production training system in the scope of intelligent factory, In: Burduk, A., Mazurkiewicz, D. (eds.), Intelligent systems in production engineering and maintenance ISPEM 2017, Advances in Intelligent Systems and Computing, Vol. 637, Springer, Cham, Switzerland, 450-458, doi: 10.1007/978-3-319-64465-3 43.

[15] Lasi, H., Fettke, P., Kemper, H.-G., Feld, T., Hoffmann, M. (2014). Industry 4.0, Business \& Information Systems Engineering, Vol. 6, 239-242, doi: 10.1007/s12599-014-0334-4.

[16] Gajsek, B., Marolt, J., Rupnik, B., Lerher, T., Sternad, M. (2019). Using maturity model and discrete-event simulation for Industry 4.0 implementation, International Journal of Simulation Modelling, Vol. 18, No. 3, 488-499, doi: 10.2507/IJSIMM18(3)489.

[17] Ahuett-Garza, H., Kurfess, T. (2018). A brief discussion on the trends of habilitating technologies for Industry 4.0 and smart manufacturing, Manufacturing Letters, Vol. 15, Part B, 60-63, doi: 10.1016/j.mfglet.2018.02.011.

[18] Zhong, R.Y., Xu, X., Klotz, E., Newman, S.T. (2017). Intelligent manufacturing in the context of Industry 4.0: A review, Engineering, Vol. 3, No. 5, 616-630, doi: 10.1016/J.ENG.2017.05.015.

[19] Shen, W., Norrie, D.H. (1999). Agent-based systems for intelligent manufacturing: A state-of-the-art survey, Knowledge and Information Systems, Vol. 1, No. 2, 129-156, doi: 10.1007/BF03325096.

[20] Hermann, M., Pentek, T., Otto, B. (2016). Design principles for Industrie 4.0 scenarios, In: Proceedings of the 49th Annual Hawaii International Conference on System Sciences HICSS 2016, Koloa, Hawaii, USA, 3928-3937, doi: 10.1109/HICSS.2016.488.

[21] Ojstersek, R., Lalic, D., Buchmeister, B. (2019). A new method for mathematical and simulation modeling interactivity: A case study in flexible job shop scheduling, Advances in Production Engineering \& Management, Vol. 14, No. 4, 435-448, doi: 10.14743/apem2019.4.339.

[22] Sethi, A.K., Sethi, S.P. (1990). Flexibility in manufacturing: A survey, International Journal of Flexible Manufacturing Systems, Vol. 2, No. 4, 289-328, doi: 10.1007/BF00186471.

[23] Krolczyk, J.B., Krolczyk, G.M., Legutko, S., Napiorkowski, J., Hloch, S., Foltys, J., Tama, E. (2015). Material flow optimization - A case study in automotive industry, Tehnički Vjesnik - Technical Gazette, Vol. 22, No. 6, 1447 1456, doi: 10.17559/TV-20141114195649.

[24] Hajduk, M., Sukop, M., Semjon, J., Jánoš, R., Varga, J., Vagaš, M. (2018). Principles of formation of flexible manufacturing systems, Tehnički Vjesnik - Technical Gazette, Vol. 25, No. 3, 649-654, doi: 10.17559/TV-201610121 $\underline{32937 .}$

[25] Terkaj, W., Tolio, T., Valente. A. (2009). A review on manufacturing flexibility, In: Tolio, T. (ed.), Design of Flexible Production Systems, Springer, Berlin, Heidelberg, Germany, 41-61, doi: 10.1007/978-3-540-85414-2_3.

[26] Terkaj, W., Tolio, T., Valente, A. (2009). Focused flexibility in production systems, In: ElMaraghy, H. (ed.), Changeable and reconfigurable manufacturing systems, Springer series in advanced manufacturing, Springer, London, United Kingdom, 47-66, doi: 10.1007/978-1-84882-067-8_3.

[27] Singh, S.P. (2014). Production and operations management, Vikas Publishing House, Noida, India.

[28] Zülch, G., Jonsson, U., Fischer, J. (2002). Hierarchical simulation of complex production systems by coupling of models, International Journal of Production Economics, Vol. 77, No. 1, 39-51, doi: 10.1016/S0925-5273(01)00 198-0.

[29] Brey, P. (2008). Virtual reality and computer simulation, In: Himma, K.E., Tavani, H.T. (eds.), The handbook of information and computer ethics, John Wiley \& Sons, New York, USA, 361-384, doi: 10.1002/97804702818 19.ch15.

[30] Yang, S.L., Xu, Z.G., Li, G.Z., Wang, J.Y. (2020). Assembly transport optimization for a reconfigurable flow shop based on a discrete event simulation, Advances in Production Engineering \& Management, Vol. 15, No. 1, 69-80, doi: 10.14743 /apem2020.1.350.

[31] Saez-Mas, A., Garcia-Sabater, J.P., Morant-Llorca, J. (2018). Using 4-layer architecture to simulate product and information flows in manufacturing systems, International Journal of Simulation Modelling, Vol. 17, No. 1, 30-41, doi: $10.2507 /$ IJSIMM17(1)408.

[32] Straka, M., Malindzakova, M., Rosova, A., Trebuna, P. (2016). The simulation model of the material flow of municipal waste recovery, Przemysl Chemiczny - Chemical Industry, Vol. 95, No. 4, 773-777, doi: 10.15199/62.2016. 4.12.

[33] Mourtzis, D., Doukas, M., Bernidaki, D. (2014). Simulation in manufacturing: Review and challenges, Procedia CIRP, Vol. 25, 213-229, doi: 10.1016/i.procir.2014.10.032. 
[34] Straka, M., Khouri, S., Lenort, R., Besta, P. (2020). Improvement of logistics in manufacturing system by the use of simulation modelling: A real industrial case study, Advances in Production Engineering \& Management, Vol. 15, No. 1, 18-30, doi: 10.14743/apem2020.1.346.

[35] Istokovic, D., Perinic, M., Dobovicek, S., Bazina, T. (2019). Simulation framework for determining the order and size of the product batches in the flow shop: A case study, Advances in Production Engineering \& Management, Vol. 14, No. 2, 166-176, doi: 10.14743/apem2019.2.319.

[36] Pedgen, C.D., Shannon, R.E., Sadowski, R.P. (1995). Introduction to simulation using SIMAN, McGraw Hill, New York, USA. 
APEM
Advances in Production Engineering \& Management Letnik 15 | Številka 4 | December 2020 | Strani 467-480 https://doi.org/10.14743/apem2020.4.379
ISSN 1854-6250

Spletna stran: apem-journal.org Izvirni znanstveni članek

\title{
Simulacijski pristop za preučevanje vpliva različnih proizvodnih tokov pri izdelavi izdelkov po meri
}

\author{
Żywicki, K. ${ }^{\mathrm{a},{ }^{*}}$, Rewers, P. ${ }^{\mathrm{a}}$ \\ aFaculty of Mechanical Engineering, Poznan University of Technology, Poznan, Poland
}

\section{POVZETEK}

Izdelava izdelkov, prilagojenih individualnim zahtevam kupcev, je nujna, če želijo podjetja učinkovito konkurirati na trgu. Proizvodnja blaga po meri postavlja nove izzive za vsa področja delovanja proizvodnih sistemov. Pri organizaciji proizvodnega toka (materiali in informacije) je treba vpeljati takšna pravila in metode, ki bodo omogočali prilagodljiv odziv na spremembe zasnove izdelkov in njihove zahteve. Članek predstavlja raziskavo, ki je bila izvedena v laboratoriju SmartFactory Tehnološke univerze v Poznanu o vplivu strukture izdelkov (prilagoditev) na realizacijo trenutnih proizvodnih naročil. Raziskava je bila izvedena z uporabo simulacijskega okolja FlexSim. Na podlagi simulacijskih eksperimentov za tri oblike organizacije proizvodnega toka $\mathrm{z}$ različnimi stopnjami prilagodljivosti proizvodnih virov je bila narejena analiza časa izvedbe različnih sklopov proizvodnih naročil in stopnje izrabe razpoložljivega delovnega časa. Rezultati raziskave kažejo, da je pri proizvodnji izdelkov z nizko in visoko načrtovano porabo dela najbolj ugodna uporaba univerzalne proizvodne postaje. Za takšno rešitev je tudi stopnja izkoriščenosti razpoložljivega delovnega časa proizvodnih postaj najvišja. Ugotovljeno je bilo tudi, da načela razporejanja proizvodnih naročil vplivajo na učinkovitost proizvodnega sistema. Najboljši rezultati so bili doseženi pri proizvodnem načrtu, pri katerem je bilo zaporedje proizvodnih naročil vzpostavljeno od najkrajšega načrtovanega časa koriščenja virov.

\section{PODATKI O ČLANKU}

Ključne besede:

Pametna proizvodnja;

Proizvodni tok;

Izdelki po meri;

Variantni izdelki;

Simulacija diskretnih dogodkov

(DES);

Programska oprema za pripravo simulacije in analizo FlexSim

* Kontaktna oseba: krzysztof.zywicki@put.poznan.pl (Żywicki, K.)

Zgodovina članka:

Prejet 26. maja 2020

Popravljen 4. decembra 2020

Sprejet 7. decembra 2020 